\title{
Comunicación

\section{Sesgo partidista en medios informativos. Una crítica metodológica y propuesta}

Party bias in the media. A methodological critique and proposal

\section{MARTÍN ECHEVERRÍA ${ }^{1}$}

http://orcid.org/0000-0001-6071-8725

La constatación científica del sesgo partidista ha sido problemática dadas sus dificultades metodológicas en relación a tres aspectos aquí examinados: el estructural, cuando los investigadores naturalizan la norma de neutralidad en sistemas mediáticos donde es contingente; el organizacional, cuando se ignora la superioridad operativa de unas campañas sobre otras; y el metodológico, acerca del muestreo y validez de los indicadores usados. Las soluciones propuestas en el texto se ponen a prueba en la cobertura de las elecciones mexicanas de 2012, con resultados sugerentes.

PALABRAS CLAVE: Sesgo partidista, investigación de medios, metodología, análisis de contenido, México.

Scientific proof of party bias has been complicated due to their methodological difficulties, here examined: structural ones, when researchers take for granted the norm of objectivity in systems that could uphold different values; organizational ones, when researchers ignore the organizational superiority of some campaigns over others; and methodological ones, about sampling and validity of commonly used indicators. We suggest some practices to avoid these issues and illustrate their worth in the coverage of the Mexican presidential elections of 2012, with promising results.

KEYWORDS: Party bias, media studies, methods, content analysis, Mexico.

1 Benemérita Universidad Autónoma de Puebla, México.

Correo electrónico: echevemartin@yahoo.com.mx

Fecha de recepción: 28/10/16. Aceptación: 14/02/17. 


\section{INTRODUCCIÓN}

Como fenómeno recurrente en la relación entre medios informativos e instituciones políticas, el sesgo partidista es de las prácticas más documentadas por el campo académico de la comunicación política, la sociedad civil organizada y las autoridades electorales, respecto al desempeño de los medios informativos. Particularmente durante las elecciones el procedimiento tradicional consiste en realizar un análisis cuantitativo de contenidos a las notas publicadas por una muestra representativa de medios de comunicación. No obstante, dicho procedimiento enfrenta problemas metodológicos que dejan sin respuesta determinados aspectos de desempeño. Dos ejemplos resultan ilustrativos: en primer lugar si bien los balances finales o netos en ocasiones demuestran un desempeño imparcial de determinados medios, el análisis cualitativo y/o lingüístico pone de manifiesto algunos sesgos que pasan desapercibidos por los protocolos cuantitativos de codificación, como el uso de oraciones en voz pasiva, ironías y construcciones impersonales que adquieren así mayor fuerza (Salgado, 2009). Un segundo problema es que las muestras amplias nulifican ciertos acontecimientos de poco peso cuantitativo en la sumatoria final pero con una visibilidad e impacto públicos mayores, tales como los debates presidenciales. El balance cuantitativo neto termina por enmascarar el hecho de que esos medios, en algunos momentos altamente significativos, efectivamente desequilibran la imparcialidad de la cobertura.

Ambos casos ratifican el hecho de que, desde sus orígenes, el análisis de contenido adolezca de diversos puntos ciegos que restan fidelidad a sus mediciones, una limitación inherente a otras técnicas del paradigma positivista; aquí se sostiene que las problemáticas descritas no radican del todo en esta particularidad, sino en ciertas prácticas estandarizadas de medición que se aplican sin reparar en sus limitaciones metodológicas, y que carecen por tanto de procedimientos auxiliares que ayuden a subsanarlas. El objetivo de este ejercicio consiste en examinar los problemas que tiene el procedimiento estandarizado de análisis de contenido, por lo menos el expresado en diversos trabajos nacionales e internacionales aquí citados, para conseguir validez en sus mediciones de sesgo partidista; y se realizan algunas propuestas en forma de indi- 
cadores adicionales y procedimientos de muestreo para subsanarlos. Se presenta de igual forma un ejemplo ilustrativo de aplicación de estas sugerencias: la cobertura de la elección presidencial de 2012, que pretende demostrar sus alcances y limitaciones. En última instancia se trata de invitar a la revisión crítica de tales procedimientos en atención a su mejora, tomando distancia de la inercia y naturalidad con que estos se han arraigado en las instituciones que observan mensajes periodísticos a propósito de las elecciones.

\section{LA ASPIRACIÓN DE NEUTRALIDAD PARTIDISTA.}

FUNDAMENTOS NORMATIVOS DE BASE

En el ámbito del periodismo político se entiende por sesgo la tendencia sistemática a beneficiar a unos actores o posiciones en detrimento de otros, mediante la selección de una fuente que produce información incompleta sobre temas asociados a los mismos -la dimensión de acceso (in)equitativo - o a través de tratamientos editoriales preferenciales o bien desfavorecedores -la dimensión de tratamiento (des)equilibrado-(Fico, Freedman \& Love, 2006; McQuail, 1998). Tal práctica cobra una dimensión política importante al constituir una transgresión a las normas de imparcialidad y objetividad del desempeño periodístico, entendida este último como el equilibrio en la presentación de las diferentes facetas de un tema o acontecimiento, neutralidad en tono y forma, contención emocional, separación entre hechos y opinión, y ausencia de segundas intenciones (Christians, Glasser, McQuail \& White, 2009; McQuail, 1998; Schudson, 2001).

La transgresión de ambos principios tiene consecuencias políticas significativas desde el punto de vista democrático, ya que la representación equitativa de las opciones políticas en los medios de comunicación es una condición necesaria para procurar el derecho igualitario a la elegibilidad para la cosa pública y la consecución de elecciones competidas; este fundamento resulta imprescindible en el contexto del control ventajoso de recursos que ciertos grupos de élite tienen para expresarse en público, mismo que pudiera reducir las fuentes alternativas de información -ajenas al control de un determinado grupo económico o político-y en última instancia hacer estériles los esfuerzos de otras 
opciones políticas menos poderosas para competir (Cantú, 2013; Dahl, 2000). Por otra parte desde el punto de vista mediático normativo, la imparcialidad pretende atender el principio de diversidad según el cual los medios se conciben como escenarios que deben "reflejar" de manera equilibrada la pluralidad de los grupos e intereses sociales y políticos, en atención al interés público (McQuail, 1998). Ambos argumentos apelan a corregir desigualdades estructurales en los sistemas políticos, tan caras a la democracia.

Los elementos previamente mencionados constituyen el piso mínimo de supuestos normativos que informan las evaluaciones de desempeño periodístico elaboradas por diversos actores, de los que mencionamos la autoridad electoral, la sociedad civil organizada y la academia; estos han desarrollado procedimientos metodológicos que intentan traducir aquellos supuestos a observaciones concretas, objetivamente evaluables. En el camino dichos actores han incorporado problemas que responden, en ocasiones, a la inadecuación conceptual de los principios mencionados a los sistemas mediáticos investigados, o bien a una operacionalización insuficiente respecto al fenómeno del sesgo partidista. En la siguiente sección exploramos estos aspectos y proponemos determinadas prácticas que pueden ayudar a subsanar estos problemas.

\section{LA MEDICIÓN ACADÉMICA DEL SESGO PARTIDISTA. APRECIACIONES CRÍTICAS}

A partir de la revisión de una serie de estudios empíricos sobre sesgo partidista, Niven (2003) encuentra que a estos subyace un criterio unificado, implícito de evaluación de datos que se utiliza para sancionar la existencia y alcance de sesgo partidista, denominado por el autor "regla de proporcionalidad": en un sistema de dos (o más) partidos que produce igual número de candidatos esencialmente cualificados, y cada campaña genera eventos, actividades y discusión en cantidades casi iguales, la cobertura debe ser correspondiente con ello y cualquier desviación puede ser considerada consecuencia de un tipo de sesgo por afinidad partidista.

No obstante, este criterio de evaluación entra en tensión con las diversas condiciones en las que actúan los medios informativos, que, por 
un lado, hacen poco realista o incluso deseable esperar que se verifique una cobertura sin sesgo, y por el otro, generan una forma de medirla con limitaciones significativas (Niven, 2003). En específico oponemos tres de ellas, a manera de objeciones a dicha expectativa: una de tipo histórico cultural, en virtud de la cual la parcialidad partidista en determinados sistemas mediáticos es parte de una tradición periodística y no una transgresión; otra de tipo organizacional, referente a la habilidad diferenciada de los actores políticos para acoplarse con éxito a las rutinas de cobertura noticiosa; y otra de tipo metodológico, relativa a la manera en que se operacionaliza y mide el sesgo. Estas condiciones comprometen la validez de las evaluaciones de sesgo, cuando este se mide y atribuye en relación a una línea editorial intencionada para favorecer a un(os) partido(s) por encima de otro(s) y no a dichos aspectos.

Respecto a la primera, relativa a las circunstancias histórico culturales, cabe recordar que el atributo contrario al sesgo partidista, la imparcialidad, es una norma profesional adherida al modelo "objetivo" del periodismo, una ideología profesional anglocéntrica cuya contingencia se universaliza como expectativa dominante, central y en ocasiones esencial en diversos sistemas mediáticos (Hallin \& Mancini, 2004). En varias sociedades dicha ideología no se sostiene en la práctica, en buena medida por la inhibición histórica de un sentido de interés público (Papathanassopoulos, 2001); no obstante sus promotores en la academia, el gobierno y el propio campo periodístico, sostienen tal expectativa, sin reparar en las circunstancias endógenas que la circunscriben y en consecuencia moderan su factibilidad.

Visto de otra manera, la parcialidad partidista en diversos sistemas mediáticos es un atributo convencional e incluso deseable: sucede en el rol auto-asumido de abogacía de cierto tipo de prensa "comprometida" y abiertamente ideológico partidista, un fenómeno tenido como saludable para el buen desempeño del sistema; en el rol facilitador que, al amparo de los principios de responsabilidad social y el ejercicio del periodismo como medio para mejorar la calidad de vida, se toma la libertad de emitir sesgos en función de un criterio de interés público (Christians et al., 2009); y en los sistemas polarizados políticamente, donde la diversidad de grupos políticos antagónicos se corresponde con medios sesgados políticamente que atienden a sus propios mercados, circunstancia concep- 
tualizada como "diversidad externa“(McQuail, 1998). En estos casos los periodistas se comportan de acuerdo a una lógica cultural que favorece el sesgo partidista, pues se espera que estén comprometidos a reportar una particular selección de eventos y ofrecer una interpretación idiosincrásica, no notarial, de la realidad, aunque sin renunciar a principios elementales de profesionalismo. En esa lógica, por el contrario, ser neutral es tenido como una falta de compromiso, civilidad o lealtad política, como lo demuestra la tradición periodística de denuncia en los sistemas mediáticos francés y holandés (Hallin \& Mancini, 2004).

Como es notorio, la posición contingente del periodismo respecto a las expectativas sociales dificulta ser definitivos acerca de las normas que deberían aplicar (Christians et al., 2009). Sin embargo, muchos trabajos plantean un diseño e interpretación de los datos partiendo del supuesto normativo de que un desempeño neutral u objetivo de los medios en la cobertura electoral es un componente esencial de un sistema democrático; y que la parcialidad partidista es una disfunción del mismo, más inquietante mientras más profunda y generalizada sea. Las circunstancias previamente descritas contradicen dicha postura, pues la valoración de la imparcialidad depende del marco de condiciones reales del sistema mediático de que se trate.

Una segunda fuente de sesgo, ahora de naturaleza organizacional, deriva de los méritos, habilidades, profesionalismo y desempeño de ciertos gestores o equipos de campaña para adaptarse a los procesos y rutinas informativas (newsmaking) de los medios, en detrimento de otros equipos. Debido a sus limitaciones organizacionales para "reportar la realidad", los medios se sirven de la provisión de informaciones y eventos que los equipos de campaña ponen a su disposición, aunque en cantidad y calidad desiguales. Así, una campaña bien adaptada al newsmaking pudiera organizar eventos llamativos, planificados para la conveniencia de su reporteo; acoplar la producción de sus comunicaciones a los valores y lenguajes de los medios; y presentar candidatos más noticiables, prominentes y citables de acuerdo a sus rasgos de "personalidad mediática": afectividad, credibilidad, riqueza de ideas y experiencias (Fico et al., 2004; Niven, 2003; Shoemaker \& Reese, 1996).

En principio un mejor tratamiento de tal campaña por parte de los medios los haría parecer sesgados, cuando en realidad es su habilidad 
para generar una cobertura asequible y atractiva lo que produce una cobertura favorable; en sentido estricto el sesgo se acometería cuando los medios equilibran la cobertura a pesar de que unos actores se adapten exitosamente a su funcionamiento mientras que otros permanezcan desprofesionalizados, pasivos y poco accesibles.

De igual manera la naturaleza misma de los desequilibrios de poder en el sistema de partidos pudiera favorecer a una opción partidista en detrimento de otra; es evidente que existen diferencias en el peso político de los partidos de acuerdo a sus distintos tamaños electorales y papeles en un sistema político (de mayorías, minorías, de temas, etc.) que bajo el principio de jerarquía, generan niveles de noticiabilidad diferenciados (Hopmann, Van Aelst \& Legnante, 2012). Asimismo, en ocasiones ciertos acontecimientos en los que se ve involucrado un candidato tienen mayor cobertura, tales como las declaraciones accidentales, pifias, manifestaciones, etc., en función de valores de noticiabilidad que prescriben mayor atención. En síntesis, puede ocurrir que el sesgo hacia una u otra facción refleje los desequilibrios de poder y la naturaleza accidentada de los acontecimientos de campaña, que termina por inclinar involuntariamente la balanza hacia alguna de ellas; por consiguiente, pudieran resultar espurias las evaluaciones que no distinguen entre las diferencias de cobertura posiblemente atribuibles a un sesgo partidista, y las que se deben al desempeño superior de un partido en función de sus méritos y el acoplamiento exitoso al newsmaking (Schiffer, 2006).

Un último problema general de los tres explorados remite a los supuestos y procedimientos epistémico-metodológicos comúnmente utilizados para medir el sesgo, en particular tres de ellos. Tal vez el más sencillo y a la vez complejo es en relación a la categoría de "tono" de las notas o su valencia, en muchos casos el único indicador de tratamiento editorial, típicamente clasificado como positivo o negativo a partir de los adjetivos presentes en las notas; en razón de dicha operacionalización el procedimiento detecta más bien sesgos explícitos, desatendiendo aquellos latentes que emplean estrategias discursivas sofisticadas, como las mencionadas en la introducción (Salgado, 2009). Por otro lado huelga decir que varios trabajos no reportan medidas de fiabilidad para dicha variable o cuando lo hacen, no las consiguen (Fico, Zeldes \& Diddi, 2004). 
Otro problema metodológico generalizado, pero no por ello menos inquietante, atañe al hecho de que se le asigna el mismo valor cualitativo a todas las piezas del corpus. En términos de inferencias concluyentes, al agregar los casos se equipara la significatividad e impacto potencial de los acontecimientos cubiertos - de extrema variación-y se produce la impresión de que las líneas editoriales que preceden a dichos tratamientos, ya sesgados, ya neutrales, son consistentes para todos los acontecimientos por igual, dándole "el mismo peso a hechos distintos" (Graber \& Dunaway, 2015, p. 353). En efecto, ciertos medios pudieran efectuar sesgos que, aunque esporádicos y por lo tanto irrelevantes a nivel cuantitativo, serían realizados pensando que su impacto en la audiencia será significativo. Un ejemplo serían los debates televisados. A sabiendas de que tales acontecimientos atraerán mayor audiencia, un medio puede darle mejor visibilidad y tratamiento a favor de un partido, pero tal sesgo es compensado subsecuentemente con cobertura equitativa en eventos de poco impacto público de parte del candidato contrario. Sin una ponderación del impacto del acontecimiento, el análisis cuantitativo sumario concluirá espuriamente que existe imparcialidad en la cobertura.

Por último, son también problemáticas las conclusiones a las que arriban varios estudios. Desde su origen la técnica de análisis de contenido se propuso con una doble finalidad: una de tipo descriptiva y otra de tipo interpretativa, en la medida que permite elaborar inferencias hacia las intenciones de los emisores (Riffe, Lacy \& Fico, 1998). El problema en diversos trabajos sobreviene cuando se realiza una valoración llana de los datos y se infiere a partir de ellos una política editorial "alineada" de los medios, sin confrontarlos, por ejemplo, a la regulación vigente respecto a equidad de medios, que habría que explicitar, o bien tomar en consideración el éxito que tiene un candidato para acoplarse al newsmaking y ganar con ello mejor cobertura (Groeling, 2008).

\section{ALGUNAS PROPUESTAS METODOLÓGICAS} PARA LOS PROBLEMAS EXAMINADOS

A propósito de estos problemas, es posible plantear algunas salidas lógicas y otras sugeridas por la literatura. En primer lugar, implementar 
mediciones sofisticadas y complementarias a la codificación preferente de "tono" o valencia, poniéndose al día respecto a la creciente sofisticación de los medios para acometer sesgo. Una de ellas es la elaboración de un índice que sintetice indicadores estructurales acerca de la colocación de los candidatos en las notas: el orden en que se manifiestan, el tiempo o espacio que se merecen sus aserciones, la manifestación de citas directas de los mismos, entre otros, (Fico et al., 2004), algunos de ellos ya utilizados en ciertos monitoreos, como el oficial de México en 2012; o bien identificar en las notas la presencia de la agenda de un partido, demostrando convergencia entre ésta y la del medio (Graber \& Dunaway, 2015). Otra manera de sofisticar la medición implica realizar ejercicios longitudinales que muestren patrones de consistencia de sesgo en el sistema de medios (Hopmann et al., 2012), puesto que las mediciones transversales habituales pudieran estar asociadas a coyunturas de campañas mediáticamente atractivas, susceptibles de producir sesgos estructurales antes que partidistas.

Por otro lado también sería recomendable combinar los censos o muestreos que se realizan sobre la cobertura periodística con procedimientos de muestreo en función de "líneas de base", esto es, acontecimientos o condiciones relativamente objetivas, de una interpretación cultural más o menos consensuada, o línea de base -como en las cifras de homicidio, por ejemplo- de modo que sea posible esperar de manera realista una determinada cobertura (Schiffer, 2006); si ocurre alguna distorsión en el tratamiento es posible atribuirla a un sesgo partidista más que al proceso de newsmaking.

Asimismo es deseable introducir dos consideraciones en los veredictos concluyentes sobre sesgo. En primer lugar, plantear expectativas e interpretaciones de desempeño de medios matizadas por la tradición periodística y el sistema mediático en donde actúan, de manera que aquellas tengan un origen endógeno. Eso se traduciría, por ejemplo, en considerar como satisfactorio un resultado final de diversidad externa -sesgos individuales por medio, equilibrio en el conjunto de los mismos- en lugar de privilegiar a la diversidad interna -equilibro al interior de cada medio- como el criterio adecuado de desempeño editorial. En esa dirección se requiere reorientar la evaluación de los datos de un nivel individual, que analiza de manera singular o agregada a los me- 
dios, a nivel de sistema mediático con sus particulares características, tradiciones y contradicciones.

En segundo lugar, se requiere tomar en cuenta el problema del peso simbólico de los partidos en el escenario político así como los méritos de desempeño de una campaña por sobre otras, que relativicen la incidencia de una línea editorial partidista al considerar también la habilidad, profesionalismo y "músculo" propagandístico de los partidos. En esa lógica sería posible concluir concluir el partidista cuando los datos evidencian que los medios no utilizan criterios periodísticos de noticiabilidad al cubrir la política, tales como el estatus y relevancia de los partidos y actores, inferidos por la proporción de escaños en los parlamentos o los resultados de la elección, su posición en las encuestas, que hace más prominente a ciertos actores vía cobertura horse race (competencia de posiciones en las encuestas), el carisma y popularidad de los candidatos, o la atención a aspectos conflictuales en lugar de consensuales (Hopmann et al., 2012). Si estos patrones están presentes de manera proporcional en las distintas opciones políticas, pero la cobertura está inclinada claramente en favor de alguna de ellas, sería posible concluir que se suscita un sesgo editorial por afinidad partidista.

Dicha evaluación implica una práctica científica tendiente a hacer inferencias con cautela y de manera multifactorial, evitando la confusión entre los sesgos inherentes a la dinámica del campo periodístico, de naturaleza estructural, y aquellos voluntarios de origen ideológico, partidista y/o clientelar.

\section{LAS SOLUCIONES A PRUEBA.}

BREVE EJERCICIO METODOLÓGICO SOBRE EL CASO MEXICANO

El ejercicio que se presenta a continuación pretende demostrar la conveniencia metodológica de implementar estrategias capaces de atenuar los problemas examinados previamente, poniendo a prueba las sugerencias expuestas. El escenario ilustrativo es la cobertura periodística de la elección presidencial de 2012 en México, cuyo contexto histórico -expuesto para valorar los datos- se expone a continuación.

Desde hace varios lustros se ha manifestado en México una alta sensibilidad hacia el fenómeno del sesgo partidista, debido al papel 
histórico e integral que jugaron los medios durante décadas en favor del régimen autoritario y la hegemonía del partido de Estado (Partido Revolucionario Institucional [PRI]), una relación caracterizada por el control de este sobre los mismos vía concesiones cuasi monopólicas, publicidad oficial y sobornos. Aunque el sistema se fue resquebrajando paulatinamente para dar paso a coberturas cada vez más plurales (Lozano, 2001), existe todavía una impronta de recelo respecto al desempeño de los medios frente al poder, por buenas razones: a pesar de su acelerada transición, el sistema mediático mexicano aún puede caracterizarse como "liberal capturado" (Guerrero \& Marquez, 2014), dado que legal y formalmente se proclama adherido a las normas del modelo libertario, neutral y objetivo, como lo constatan las prescripciones de pluralidad, objetividad, imparcialidad y veracidad contenidas en la Constitución Mexicana (Art. $6^{\circ}$ ) y la Ley Federal de Telecomunicaciones y Radiodifusión (Art. 1), ${ }^{2}$ pero que en términos empíricamente verificados, a nivel nacional y en diversas localidades, reproduce una cultura periodística autoritaria que inercialmente prolonga la subordinación editorial de los medios a los actores políticos mediante una mercantilización clientelar de sus espacios (Aceves, 2010; Andrade, 2012; de León, 2012; Espi-

2 Dicho artículo establece que "toda persona tiene derecho al libre acceso a información plural y oportuna", que preserve además la "veracidad de la información"; el Artículo 1 de la Ley Federal de Telecomunicaciones y Radiodifusión aboga por la "difusión de información imparcial, objeti$v a$, oportuna y veraz del acontecer nacional"; adicionalmente en las recomendaciones de cobertura noticiosa que el INE envía a los concesionarios se refrendan los principios de imparcialidad y equidad en términos de un tratamiento homogéneo en cuanto a número de reportajes, entrevistas, representantes partidistas y debates, una presentación "imparcial, neutral y objetiva" de las noticias incluso en términos de los recursos audiovisuales utilizados, y una clara separación entre información y opinión (Artículos 184 la Ley General de Instituciones y Procedimientos Electorales y 6 del Reglamento de Radio y Televisión del Instituto Nacional Electoral). En todas estas recomendaciones subyace el modelo anglo americano de periodismo neutral y objetivo, sin consideración de otros modelos más ideologizados y por lo tanto parciales o interesados políticamente. 
no \& Mendoza, 2015; Gonzalez, 2013; Guerrero \& Marquez, 2014; Ortiz \& Gómez, 2013).

Una implicación de estas condiciones es que la norma de objetividad que se sostiene es más idealista que práctica (Schudson, 2001), reforzada desde las escuelas de periodismo (Hernández, 2004), ciertos círculos de periodistas (Marquez, 2010) y los discursos gubernamental y mediático corporativo, estos últimos en buena medida para enfatizar una condición de autonomía periodística de cara a audiencias escépticas. Al convivir con una tradición crítica y comprometida de periodismo, si bien minoritaria, la adherencia de los periodistas a las normas periodísticas resulta difusa y contradictoria; en esas condiciones la evaluación del desempeño periodístico se vuelve problemática y genera tensiones entre los involucrados (audiencias, propietarios, reguladores, políticos), lo que hace prudente reconsiderar un uso dominante del criterio de neutralidad para adoptar parámetros de evaluación circunstanciados, acordes a las tradiciones periodísticas vigentes.

Por su parte, la problemática histórica y contemporánea previamente descrita ha derivado, por lo menos desde 1988, en el establecimiento de ejercicios rutinarios de análisis de contenido de la cobertura electoral por parte de la academia, la sociedad civil y la autoridad electoral, de considerable envergadura, creciente sofisticación y vitalidad. En la elección de 2012 se cuentan cuatro organizaciones civiles y quince observatorios universitarios (Buendía, 2013); no obstante estos ejercicios configuran determinadas comprensiones, costumbres e inercias operativas, en la forma de medir el fenómeno, que implican varios de los problemas aquí descritos.

Tomando estos elementos como contexto de interpretación, se elaboró un análisis de contenido que incorpora las siguientes prácticas complementarias a las habituales de un monitoreo de sesgo partidista: se integró una muestra por conveniencia basada en el criterio de líneas de base y cobertura esperada (Lowry, 2008; Schiffer, 2006). Esto implica conformar la muestra de ciertos hechos cuya naturaleza más o menos predecible pudiera revelar con mayor facilidad una posición editorial determinada; así, fueron seleccionados acontecimientos con una cualidad agonal (los dos debates televisados), políticamente significativos (inicio y cierre de campaña) o patentemente negativos para 
algún candidato (multas de la autoridad electoral hacia los partidos, el conflicto del candidato del PRI, Enrique Peña Nieto, en una Universidad y las marchas que suscitaron posteriormente, así como un escándalo de recaudación ilegal de fondos conocido como "charolazo"; 3 bajo el supuesto de que dichas cualidades los hacen más susceptibles de sesgo partidista. Por razones de disponibilidad, este corpus fue extraído de siete rotativos de circulación nacional (El Universal, Excélsior, La Crónica, La Jornada, La Prensa, Milenio y Reforma), resultando analizadas 225 notas del género informativo (las piezas de opinión se excluyen en función de que naturalmente y de manera legítima manifiestan sesgos editoriales, por lo que quedan fuera de los problemas tratados en este texto).

Adicionalmente al indicador convencional de "tono" o valencia de las notas, ${ }^{4}$ se insertaron en el libro de códigos tres indicadores de tipo estructural: el balance, que constata que hay más de un partido o actor involucrado en la nota y por lo tanto una rendición equilibrada de puntos

3 Respecto al primer acontecimiento, el mencionado candidato acudió a un foro en las instalaciones de la Universidad Iberoamericana -institución privada de renombre en México- en el que enfrentó severas críticas y abucheos por parte de su comunidad estudiantil. Su partida súbita y bochornosa en respuesta a ello acrecentó la sospecha de que había franjas importantes de la población, sobre todo juvenil, que manifestaban profundo rechazo a su candidatura. Finalmente, la cobertura desfavorable hacia los estudiantes de parte de varios medios de comunicación detonó el movimiento cívico \#YoSoy132, en contra de la parcialidad periodística de los medios afines al PRI. Por lo que toca al "Charolazo" se trata de un escándalo de financiamiento ilegal de una campaña; durante la contienda un periódico difundió audios en los que un colaborador cercano al candidato izquierdista Andrés Manuel López Obrador pedía dinero a un grupo de prominentes empresarios para poder ganar la elección, una forma mal vista de recaudación; este acontecimiento dañaba uno de los ejes campaña de dicho candidato, construido alrededor de su integridad.

4 Definido como "cuando el medio (y no la fuente) utiliza explícitamente adjetivos y expresiones usadas como adjetivos" (IFE, 2012b, p. 11) que muestran a los actores en una luz positiva, negativa o neutra. 
de vista (Carter, Fico \& McCabe, 2002); la editorialización, que verifica la interpretación acerca del valor y significado de los acontecimientos en espacios tradicionalmente proscritos para ello, como las notas (Niven, 2003); y el del actor principal declarado, cuando el adversario del actor del que trata la nota se coloca al principio de la misma y adquiere mayor visibilidad y actúa como una suerte de definidor primario (Fico et al., 2004). Además se retoma de Fico et.al. (2004) la propuesta de un índice 5 que conjunte los cuatro indicadores se comparó con el de tono; las prácticas incorporadas ponen de manifiesto la diferencia, mayor o menor, que se evidencia cuando se usan varios indicadores condensados a diferencia de solo uno. En términos del índice construido los indicadores fueron transformados como sigue:

$$
Y=\sum_{i=1}^{n} X_{i}
$$

Donde: $Y=$ resultado del índice, $n=$ Número de indicadores, $i=$ indicador, $\mathrm{y} X_{i}=$ valor normalizado del indicador.

5 A partir de la literatura de geografía social, un índice se define como "un signo que resume información relevante de un fenómeno particular [...] e incluye variables que son una representación operacional de un atributo, tal como una cualidad y/o característica de un sistema" (Birkmann, 2007, p. 57). Incluye los procedimientos estadísticos de normalización (unificar las variables con diferentes unidades de medida en una sola), ponderación (asignar un peso cuantitativo a una variable si se considera que tiene un peso cualitativo diferenciado) y agregación de los indicadores por media aritmética o bien geométrica (Rygel, O’Sullivan \& Yarnal, 2006). Bajo el principio de parsimonia, la construcción del índice propuesto implicó la conversión de los valores dicotómicos en una escala de 0 a 1 , la no ponderación y la agregación por media aritmética simple. El resultado se obtiene al promediar los distintos indicadores utilizados una vez que cada uno de ellos ha sido normalizado, esto es, convertido a valores de 1 o 0 dependiendo por lo general de la ausencia (0) o presencia (1) de un cierto atributo (como los de negatividad, editorialización o declaración de actor, que se proponen para este ejemplo) 
Esta propuesta exploratoria tiene dos delimitaciones: no introduce el indicador de visibilidad de las notas al interior de las publicaciones, $\mathrm{y}$, si bien evidencia que un tratamiento determinado resulta sesgado, no indica a favor de quién, un asunto que puede resolverse complementándolo con otras medidas. Es menester subrayar que el ejercicio empírico que se propone a continuación no es un estudio de caso, dígase, no tiene el cometido de investigar las prácticas de sesgo en la elección de 2012 ni agotar la totalidad de indicadores; tan sólo sirve como ejemplo ilustrativo acerca de las ventajas de recurrir a estrategias de muestreo y síntesis de indicadores que complementen los procedimientos tradicionales.

\section{HALLAZGOS}

Una primera lectura remite a las incidencias diferenciadas de sesgo en los distintos acontecimientos elegidos para la muestra no probabilística. En su conjunto todos los acontecimientos son poco sesgados (0.31) pero algunos de ellos se distancian de esta media tales como los cierres de campaña (0.43), eventos de fuerte carga simbólica para los candidatos de cara a la elección, el segundo debate (0.39) con apreciaciones de tipo ganar o perder y el suceso del candidato del PRI en la Universidad Iberoamericana (0.38), que a la luz de las cifras se presupone desató tratamientos intencionados de parte de los medios. En segundo orden se encuentra la cobertura del evento de inicio de campaña de los candidatos (0.32) y el primer debate televisado (0.32); en último lugar están la llamada "marcha anti Peña" (0.27), el escándalo del "Charolazo" $(0.21)$ y las multas del IFE (0.17) que se sesgan poco. La señalada distancia entre este último acontecimiento y los cierres de campaña, de 26 puntos en una escala de 100, muestra criterios editoriales ampliamente diferenciados en la cobertura de distintos acontecimientos, en particular aquellos que tienen mayor impacto público o carga simbólica, como los debates y cierres de campaña, y en consecuencia del efecto de enmascaramiento que puede ocurrir cuando sesgos cualitativamente significativos se hacen equivalentes para condensarse en una sumatoria final. En general, al valorar el sesgo por tipo de acontecimiento, los originados en eventos negativos o polémicos paradójicamente son los más neutra- 
les (0.28), en comparación con los debates (0.34), poco sesgados, y los eventos de apertura y cierre de campaña, los más (0.37).

Un segundo beneficio de las estrategias metodológicas propuestas consiste en obtener una medida complementaria, más sintética, de cobertura distorsionada hacia los candidatos. Una primera observación relevante es que el candidato Gabriel Quadri fue el que recibió una cobertura más sesgada en los acontecimientos elegidos en la muestra (0.38) y Andrés Manuel López Obrador el que menos (0.27). Enrique Peña Nieto (0.30) y Josefina Vázquez Mota (0.32) recibieron sesgos similares al promedio (0.32) que es razonablemente moderado. La dirección del sesgo se puede esclarecer con otros indicadores: bajo el indicador de tratamiento la cobertura en general resultó más favorable para Quadri (27\%) y Peña (10.9\%) y un tanto menos para López Obrador (9.8\%) y Vázquez Mota (4.8\%). Bajo el indicador de editorialización la neutralidad favorece a López Obrador (90.2\%), seguida de Quadri (85.7\%), Peña (70.5\%) y Vázquez Mota (71.4\%). La ausencia de balance, una presentación que beneficia a los actores al eludir en la nota a algún opositor, señala a Quadri (78.6\%), seguido de Vázquez Mota (57.1\%), Peña (48.4\%) y López Obrador (45.1\%). Si se contempla desde cada indicador aislado, se generarían lecturas distintas acerca de qué opción política fue más sesgada; en cambio, la combinación de los indicadores en un índice, los relativiza al tiempo que equilibra el juicio acerca del sesgo acometido.

No obstante, el indicador más revelador pudiera consistir en el índice de sesgo de los candidatos en relación a los eventos más significativos de la campaña. En el primer debate la cobertura más neutral corresponde a Peña (0.25), seguido de López Obrador (0.31), Vázquez Mota (0.36) y finalmente Quadri (0.40), con un grado considerable de distorsión. En el cierre de campaña, otro acontecimiento de fuerte carga simbólica, se repite el mismo orden: Peña es el actor que se reporta de manera más neutral (0.33), seguido de López Obrador (0.43), Quadri (0.50) y Vázquez Mota (0.56). De manera interesante la llamada "marcha anti Peña" le generó cobertura neutral a los actores, con un sesgo insignificante tanto para Peña (0.17) como para López Obrador (0.13), neutralidad total en las declaraciones de Vázquez Mota y ningún pronunciamiento publicado de parte de Quadri. En este sentido y al menos 
en estos tres acontecimientos, Peña fue favorecido no con una cobertura favorable, sino con la neutralidad en el reporteo de sus declaraciones.

Por su parte, los rangos de sesgo por rotativo son muy variables. Hay medios con un comportamiento muy poco (Excélsior, 0.11), poco (El Universal, 0.21, La Prensa, 0.31 y La Crónica, 0.34) o bien considerablemente sesgado (Reforma, 0.41, La Jornada, 0.43); el primero y La Crónica no orientaron sesgo hacia algún candidato, mientras que los candidatos fueron favorecidos de manera diferenciada en los distintos periódicos: Quadri con el 25\% de notas favorables en El Universal y La Prensa, López Obrador con el 50\% y 75\% de notas favorables en Reforma y La Jornada, respectivamente, y Peña con el 40\% de notas favorables en La Prensa.

Por último, es necesario contemplar cómo se ha de evaluar el desempeño de los medios respecto a estos datos de sesgo. Si procedemos bajo una sanción llana de origen regulatorio es evidente que los medios incumplen las prescripciones constitucionales de objetividad, imparcialidad y veracidad; mas, la vaguedad y falta de operacionalización de estos principios dificulta un examen preciso de desviación a la norma. Un segundo criterio implica juzgar el desempeño de los medios de manera relativa, tomando algunos indicadores de desempeño de los partidos y campañas que pudieran revelar condiciones objetivas susceptibles de provocar sesgo involuntario o bien un acoplamiento exitoso de las mismas a las rutinas periodísticas, en términos de noticiabilidad. Tres aspectos se toman en cuenta respecto a estos condicionamientos: el diferencial de financiamiento de cada campaña, que pudiera traducirse en una mayor profesionalización y capacidad de organización de eventos mediáticos; la composición de la Cámara de Diputados al momento de las elecciones, que remite al peso político que los periodistas interpretan tiene cada partido en el espacio público (principio de jerarquía); y los resultados finales de la elección, que indican aproximadamente los candidatos que parecían "ganadores" a los ojos de los periodistas y por lo tanto más noticiables (lógica de "carrera de caballos"). En la siguiente Tabla presentamos datos relativos a los partidos en contienda en relación a dichos indicadores.

La Tabla condensa un diferencial importante en la posición y recursos de los partidos: la alianza PRI/PVEM se revela, por mucho, como 
TABLA 1

INDICADORES DE PROMINENCIA PARTIDISTA

EN LAS CAMPAÑAS ELECTORALES

\begin{tabular}{lcrrrrrr}
\hline \multirow{2}{*}{$\begin{array}{c}\text { Alianzas } \\
\text { partidistas en } \\
\text { campaña }\end{array}$} & \multicolumn{2}{c}{$\begin{array}{c}\text { Financiamiento de } \\
\text { campaña }\end{array}$} & \multicolumn{3}{c}{$\begin{array}{c}\text { Escaños en el } \\
\text { Congreso }\end{array}$} & \multicolumn{2}{c}{$\begin{array}{c}\text { Resultados de la } \\
\text { elección }\end{array}$} \\
\cline { 2 - 8 } & $\begin{array}{c}\text { Recursos } \\
\text { (en pesos) }\end{array}$ & $\%$ & Escaños & $\%$ & Votos & $\%$ \\
\hline PRI / PVEM & 693776955 & 41.3 & 256 & 52.4 & 19226784 & 39.2 \\
PAN & 424784163 & 25.3 & 142 & 29.0 & 12786647 & 26.1 \\
PRD / PT / MC & 446903630 & 26.6 & 83 & 17.0 & 15896999 & 32.4 \\
Nueva Alianza & 115095669 & 6.8 & 8 & 1.6 & 1150662 & 2.3 \\
\hline
\end{tabular}

Fuente: Elaboración propia con base en datos del Instituto Federal Electoral (IFE, 2012a).

la más prominente en el Congreso ( $52 \%$ de escaños) lo que trae como consecuencia indirecta un mayor poder de establecimiento de agenda; es también el que recibió mayor financiamiento de campaña, traducido en una mayor visibilidad pública vía propaganda; y resultó un claro ganador en la elección. En ese orden de ideas, el PAN se encuentra en segunda posición respecto al número de escaños $(29 \%)$, pero recibe un financiamiento similar (25\%) a la coalición de izquierda, PRD, PT y $\mathrm{MC}$, con el $26 \%$, aunque queda en tercer lugar en los resultados finales (26\%) superado considerablemente por la coalición de izquierda (32\%). La jerarquía y recursos políticos de acuerdo a estos indicadores claramente muestran al PRI/PVEM en primera posición, seguido del PRD/PT/ MC y PAN en última instancia.

Si las rutinas periodísticas son sensibles a eventos mediáticos y un ambiente propagandístico intenso, ambos vinculados a un financiamiento generoso; a una jerarquía de partidos donde unos son más prominentes para la opinión pública que otros, y por tanto más noticiables; y tienden a favorecer a candidatos "punteros" bajo narrativas horse race, es posible argumentar que la falta de neutralidad y tendenciosidad de los medios a favor del candidato del PRI parece asociada a factores objetivos de influencia política en las rutinas periodísticas, 
más que a un sesgo editorial de tinte partidista. Finalmente, una interpretación poco consensuada entre los periodistas acerca del valor de la neutralidad política termina configurando un escenario de diversidad externa, en el que los sesgos parciales por medio se nulifican entre sí. En suma, a partir de los casos examinados, con el uso de este diseño metodológico en particular y en relación a los criterios de evaluación puestos en práctica, se puede concluir que el desempeño de la prensa en la cobertura de las elecciones de 2012 no tiene un sesgo partidista pronunciado, un hallazgo contrapuesto a la opinión generalizada y a otros trabajos (Cantú, 2013; Martínez, 2013; Ortiz \& Gómez, 2013) que persiguen la misma finalidad pero con los procedimientos metodológicos típicos. Este resultado en conjunción con la forma de obtenerlo constituye un contrafactual que demuestra el valor distintivo de la propuesta metodológica presentada.

\section{PALABRAS FINALES}

La desconfianza ciudadana en las instituciones democráticas, incluidos los medios informativos, hacen presuponer la instrumentalización de los mismos por parte de los partidos, como meras "correas de transmisión" de las élites en turno. En efecto, los medios inclinan las coberturas periodísticas de elección en elección y transgreden determinados principios de objetividad e imparcialidad altamente estimados por diversos actores sociales así como los medios mismos; aunque eso se intenta demostrar, ello se relaciona con factores más complejos que una mera identificación partidista o la fuerza de coerción clientelar.

El presente trabajo ilustra la complejidad del fenómeno del sesgo partidista e invita a reflexionar críticamente acerca de la conveniencia de las estrategias metodológicas habituales para medirlo y evaluarlo. El procedimiento aquí vertido es desde luego perfectible y pudiera adquirir un mayor alcance aplicándolo en periodos no electorales, pero es ante todo un pronunciamiento por un debate teórico metodológico necesario a la luz de la centralidad que los medios tienen en los procesos políticos, y la necesidad recurrente de la sociedad civil y el Estado de vigilar su desempeño. 


\section{Referencias bibliográficas}

Aceves, F. (2010). La democracia no pasa por las pantallas: desigualdad, desequilibrio y ausencia de pluralismo en la cobertura informativa de las elecciones de 2009 en Jalisco. Quorum Académico, 7 (2), 57-74.

Andrade, P. (2012). Las elecciones 2010 en Veracruz y el comportamiento de la prensa. Razón y Palabra, 17 (79).

Birkmann, J. (2007). Indicators and criteria for measuring vulnerability: Theoretical bases and requirements. En J. Birkmann (Ed.), Vulnerability to Natural Hazards: towards disaster resilient societies. New York: United Nations University Press, The Energy and Resources Institute

Buendía, J. (2013). Observación electoral y medios de comunicación en México. Lecciones aprendidas y visión de futuro. Revista Mexicana de Derecho Electoral, (4), 75-101.

Cantú, J. (2013). Las televisoras vulneran la equidad en la contienda. Desacatos, 42, 79-102.

Carter, S., Fico, F. \& McCabe, J. (2002). Partisan and Structural Balance in Local Television Election Coverage. Journalism and Mass Communication Quarterly, 79, 41-53.

Christians, C. G., Glasser, T., McQuail, D. \& White, R. (2009). Normative Theories of the Media. Journalism in Democratic Societies. Illinoils: University of Illinoils Press.

Dahl, R. (2000). On Democracy. New Haven: Yale Nota Bene.

de León, S. (2012). Comunicación Pública y Transición Política. Los rasgos de lo global en el periodismo local. Un estudio situado. Aguascalientes: Universidad Autónoma de Aguascalientes.

Espino, G. \& Mendoza, E. (2015). Los gobernadores, enclaves del autoritarismo en México. Sometimiento y subordinación de los medios de comunicación locales. México: Fontamara.

Fico, F., Freedman, E. \& Love, B. (2006). Partisan and Structural Balance in Newspaper Coverage of US Senate Races in 2004 with Female Nominees. Journalism and Mass Communication Quarterly, 83(1), 43-57.

Fico, F., Zeldes, G. A. \& Diddi, A. (2004). Partisan and Structural Balance of Local Television Election Coverage of Icumbent and Open 
Gubernatorial Elections. Journalism and Mass Communication Quarterly, 81(4), 897-910.

Gonzalez, R. (2013). Economically-Driven Partisanship-Official Advertising and Political Coverage in Mexico: The Case of Morelia. Journalism and Mass Communication, 3(1), 14-33.

Graber, D. \& Dunaway, J. (2015). Mass media and american politics. Thousand Oaks: Sage, CQ Press.

Groeling, T. (2008). Who's the Fairest of them All? An Empirical Test for Partisan Bias on ABC, CBS, NBC and Fox News. Presidential Studies Quarterly, 38(4), 631-657.

Guerrero, M. A. \& Marquez, M. (2014). The "Captured-Liberal" Model: Media Systems, Journalism and Communication Policies in Latin America. En M. A. Guerrero \& M. Marquez (Eds.), Media Systems and Communication Policies in Latin America. New York: Palgrave McMillan.

Hallin, D. C. \& Mancini, P. (2004). Comparing Media Systems. Three Models of Media and Politics. Cambridge: Cambridge University Press.

Hernández, M. E. (2004). La formación universitaria de periodistas en México. Comunicación y Sociedad, 1, 100-138.

Hopmann, D. N., Van Aelst, P. \& Legnante, G. (2012). Political balance in the news: A review of concepts, operationalizations and key findings. Journalism, 13(2), 240-257.

Instituto Federal Electoral-IFE. (2012a). Cifras relevantes del proceso electoral federal 2011-2012. Recuperado el 26 de junio de 2016 de de http://www.ine.mx/

Instituto Federal Electoral-IFE. (2012b). Monitoreo de Espacios Noticiosos en Radio y Televisión Campaña Electoral para Presidente de la República 2011-2012. Recuperado el 26 de junio de 2016 de http://www2.ine.mx/

Lowry, D. T. (2008). Network TV news framing of good vs. bad economic news under democrat and republican presidents: a lexical analysis of political bias. Journalism \& Mass Communication Quarterly, 85(3), 483-498.

Lozano, J. C. (2001). Espectacularización en la cobertura informativa de las elecciones mexicanas a la Presidencia. Comunicación y Sociedad, XIV(1), 29-49. 
Marquez, M. (2010). Journalism Culture and Political Conflict: Mexican Journalists Reflect their Performance During 2006 Presidential Elections. Derecom: Revista online especializada en Derecho de la Comunicación, 1(1). Recuperado de http://www.derecom.com/blog/ item/103-journalism-culture-and-political-conflict-mexican-journalists-reflect-their-performance-during-2006-presidential-elections

Martínez, F. (2013). La contienda electoral federal 2012 en la prensa mexicana. Revista Mexicana de Opinión Pública, 15, 61-79.

McQuail, D. (1998). La acción de los medios. Buenos Aires: Amorrortu.

Niven, D. (2003). Objective evidence on media bias: Newspaper coverage of Congressoinal Party Switchers. Journalism and Mass Communication Quarterly, 80(2), 311-326.

Ortiz, M. \& Gómez, R. (2013). Una mirada a las elecciones de 2012 desde la frontera norte de México. El caso de la prensa de Baja California. Versión, estudios de comunicación y política, (32), 51-71.

Papathanassopoulos, S. (2001). Media Commercialization and Journalism in Greece. European Journal of Communication, 16(4), 505-521.

Riffe, D., Lacy, S. \& Fico, F. G. (1998). Analyzing Media Messages. Mahwah: Taylor \& Francis Group.

Rygel, L., O’Sullivan, D. \& Yarnal, B. (2006). A Method for Constructing a Social Vulnerability Index: An Application to Hurricane Storm Surges in a Developed Country. Mitigation and Adaptation Strategies for Global Change, 11(3), 741-764.

Salgado, E. (2009). ¿Qué dicen los periódicos? Reflexiones y propuestas para el análisis de la prensa escrita. México: CIESAS.

Schiffer, A. J. (2006). Assessing Partisan Bias in Political News: The Case(s) of Local Senate Election Coverage. Political Communication, 23(1), 23-39.

Schudson, M. (2001). The objectivity norm in American journalism. Journalism, 2(2), 149-170.

Shoemaker, P. \& Reese, S. (1996). Mediating the Message. Theories of Influences on Mass Media Content (2nd ed.). New York: Longman. 